\title{
Paradise revealed III: why so many ways to die? Apoptosis, necroptosis, pyroptosis, and beyond
}

\author{
Marcelo Pires Amaral (10 ${ }^{1} \cdot$ Laura Migliari Branco ${ }^{1}$ Andreas Strasser ${ }^{2,3} \cdot$ Vishva M. Dixit ${ }^{4}$. \\ Karina Ramalho Bortoluci $i^{1}$
}

Received: 20 December 2019 / Revised: 23 January 2020 / Accepted: 26 February 2020 / Published online: 17 March 2020

(c) The Author(s), under exclusive licence to ADMC Associazione Differenziamento e Morte Cellulare 2020

Paradise can be defined as a place full of beauty, happiness, joy, and enthusiasm. All of this was recently combined in an exciting scientific meeting on cell death held in Guarujá, São Paulo, Brazil, from November 19-24, 2019. The first [1] and second [2] successful Meetings held in Brazil in 2010 and 2014, respectively, opened the doors to a scientific update this year. The third Meeting on cell death "Why So Many Ways to Die? Apoptosis, Necroptosis, Pyroptosis and Beyond" was organized by KRB, AS, and VMD. Over 150 scientists from all continents, including ones from industry, healthcare professionals as well as principal investigators, postdocs, and graduate students from academia shared 5 days of intense scientific discussions and data exchange.

The Meeting happened at a particularly important moment in the field with the recent discoveries of key regulators and effectors of cell death processes and, importantly, the first drug based on cell death research, the BCL-2 inhibitor venetoclax, now being used routinely in the clinic for the treatment of certain hematological cancers. The excellent talks from prominent more experienced and young scientists were instrumental to improve our understanding of the crosstalk among distinct cell death pathways in health and disease. A fantastic and friendly atmosphere was present during the workshops, short talks, and poster

Marcelo Pires Amaral

m.piresamaral@gmail.com

1 Centro de Terapia Celular e Molecular (CTCMol), Universidade Federal de São Paulo (UNIFESP), São Paulo, Brazil

2 The Walter and Eliza Hall Institute of Medical Research, Parkville, Melbourne, VIC, Australia

3 Department of Medical Biology, University of Melbourne, Melbourne, VIC, Australia

4 Department of Physiological Chemistry, Genentech, South San Francisco, CA, USA sections, allowing new insights and prospects during discussions.

Kim Newton (Genentech, Inc., USA) presented the opening Keynote Address, which was a perfect kick-off for the event. Using mice deficient in key molecules of distinct cell death processes, Kim demonstrated that catalytically dead caspase-8 could activate caspase-1/11-dependent inflammatory responses when necroptosis was inhibited. Thus, when the protease activity of caspase- 8 and subsequent ability to induce apoptosis is attenuated by pathogen-encoded inhibitors, it can respond by driving pyroptosis. Altogether, her results demonstrated that there is much more plasticity in cell death signaling than anticipated, with caspase- 8 being a central determinant for which cell death pathway will be unleashed in a context dependent manner. This plasticity may serve to optimize immune defense against pathogens, particularly for the organism to be able to deal with pathogens that possess inhibitors of more than one mammalian cell death pathway.

\section{Advances in molecular control of cell death}

This topic started with Hao Wu (Boston Children's Hospital, USA) introducing new findings on molecular and structural biology of the NLRP3 inflammasome. She showed that "secondary" NEK7 interaction with NLRP3 is important to induce NLRP3 activation, although this is not sufficient. Since NLRP3 inflammasome activation is microtubule-dependent, there might be an aggregasomelike, microtubule-dependent mechanism for full NLRP3 activation. Veit Hornung (Ludwig-Maximilians-University Munich, Germany) demonstrated that CARD8 was capable of triggering pyroptosis in T cells independently of ASC and dependent on Gasdermin D (GSDMD). However, CARD8 activation can be curtailed by TCR activation, by a mechanism that awaits definition. Daniel Kastner (National Institute of Health, USA) demonstrated that a Pyrin 
mutation linked to the Familial Mediterranean Fever also provided resistance to Yersinia infection and a consequent better host outcome. Feng Shao (National Institute of Biological Sciences, China) talked about antitumor immunity in GSDMD-mediated pyroptosis and also roused the audience with thought provoking questions. He explained that after caspase-4 cleavage, depending on the cleavage site and fragments produced, it can become active or inactive. Shao also elucidated that most cancer cells have silenced GSDM proteins, except GSDMD. He concluded the lecture postulating that caspases do not per se determine cell fate, but that their substrates do! Eicke Latz (University of Bonn, Germany) talked about immunometabolism and bystander cell responses to pyroptosis. Finishing this section, Oliver Florey (Babraham Institute, UK) dissected entosis cell cannibalism mechanisms and its consequences. He also demonstrated the potential of a new route of entosis in regulating the fate of cancer cells.

\section{Cell death and cancer}

AS (Walter and Eliza Hall Institute of Medical Research (WEHI), Australia) discussed the small molecule compound APR-246 that was described as capable of reestablishing wild-type p53 functions in mutant p53 proteins and inducing its tumor suppression function. AS showed that APR246 is capable of killing tumor cells independent of their p53 state. He also talked about how mutant p53 sustains the growth of cancer cells and revealed that its induced loss does not in fact stop the growth of cancer cells. This indicates that drugs that could abolish mutant p53 expression are unlikely to have major impact in cancer therapy. Henning Walczak (University College London, UK) argued that blockage of TNF may not be sufficient to treat some inflammatory diseases and should be combined with other targets, such as RIPK1. Ricardo Weinlich (Hospital Israelita Albert Einstein, Brazil) revealed that the high expression of RIPK3/MLKL indicates a poor prognosis in low-grade glioblastoma patients. Ruth M. Kluck (WEHI, Australia) reminded the audience that $\mathrm{BAK}$ and $\mathrm{BAX}$ work on apoptotic cell death and described novel tool antibodies that can regulate their activity.

\section{Consequences of cell death}

VMD (Genentech, Inc., USA) presented new interesting data demonstrating that germline mutation in the tumor suppressor BAP1 resulted in cell death. A whole-genome screening using CRISPR-Cas9 revealed that the deletion of the single gene RNF2 was responsible for rescuing BAP1 mutant cells. Nevertheless, he also showed that depending on the cell type and its apoptotic gene expression, it could induce cell death, or not. Altogether, he demonstrated that mutations in tumor suppressors like BAP1 might not always result in aberrant cell proliferation; it all depends on the gene expression profile of the cell. Jonathan C. Kagan (Boston Children's Hospital, USA) showed that hyperactive dendritic cells are alive and robust producers of inflammatory mediators capable of inducing $\mathrm{T}$ cell-mediated protective immunity. Hyperactivation stimuli are uniquely capable of inducing stronger cytotoxic $\mathrm{T}$ cells and a Th1 biased response. He concluded that the lecture talking about the dual role of inflammasomes in inducing pyroptosis for cell intrinsic defense and inflammation or inducing cell hyperactivation for robust adaptive immunity. John Silke (WEHI, Australia) supported the idea that there is a hierarchy of preferred cell death responses, where necroptosis is the last. Using a caspase-8 cleavage-resistant Ripk1D325A mutant mouse strain, he showed the relevance of caspasemediated RIPK1 cleavage during embryonic development. Loss of RIPK1 cleavage favors RIPK3 TNF-dependent necroptosis and apoptosis induction. In humans, mutations that prevent caspase- 8 mediated cleavage of RIPK1 cause an autoinflammatory disease.

\section{Cell death and host defense}

Petr Broz (University of Lausanne, Switzerland) showed that Guanylate Binding Proteins (GBPs) control human noncanonical inflammasome. GBPs are capable of targeting bacteria but do not directly lyse them. Instead, they recruit caspase-4 resulting in the cell death signaling cascade dependent on GSDMD. Ricardo T. Gazzinelli (Oswaldo Cruz Foundation, Brazil) demonstrated that mice doubledeficient for caspase-1 and caspase-8 were resistant to experimental cerebral malaria. He proposed that lethality of mice was attributed not only to the presence of cleaved GSDMD and mature IL- $1 \beta$ but also to TNF- $\alpha$ expression induced by caspase-8. Russell E. Vance (University of California, USA) presented his findings about peculiarities between molecular activation of NAIP/NLRC4 and NLRP1 inflammasomes. While NAIP detects specific bacterial components such as flagellin by directly binding and followed by NLRC4 recruitment, NLRP1 acts as a "decoy" sensor of pathogen-encoded enzymes that is activated by the direct cleavage on the proteasome. KRB (Federal University of São Paulo, Brazil) discussed the requirement of NAIP and NLRC4 proteins for the killing of Trypanosoma cruzi and Zika virus by macrophages and glial cells from the central nervous system. Altogether, her data demonstrated that NAIP/NLRC4 inflammasomes operate beyond bacterial infections in a variety of cell types. Edward A. Miao (University of North Carolina at Chapel Hill, USA) talked about 
the unique role of caspase-7 during bacterial infections that could not be compensated by other caspases. In fact, caspase7-deficient mice are susceptible to Chromobacterium violaceum and Listeria monocytogenes infections even in the presence of active caspase-3. Closing this section, Dario S. Zamboni (University of São Paulo, Brazil) demonstrated that some strains of Leishmania that harbors an endosymbiotic RNA virus (LRV) induce a more severe form of the Leishmaniasis disease. Briefly, he showed that the LRV could activate TLR-3 and subvert the NLRP3-mediated response via autophagy machinery activation.

\section{Manipulating cell death for therapeutic intervention}

Andrew W. Roberts (WEHI, Australia) reinforced that venetoclax acts independently of p53 and also that BCL-2 is not the only target to induce apoptosis in cancer cells, indicating that drugs targeting other pro-survival BCL-2 family members, such as MCL-1, can be developed. Wenlin Shao (AstraZeneca, USA) demonstrated that targeting multiple pro-survival proteins can induce more effective responses and pointed out the possibility to combine multiple drugs to achieve tumor regression. Domagoj Vucic (Genentech, Inc., USA) showed that inhibition of RIPK1 reduces the severity of arthritis, skin inflammation caused by Sharpin mutations, and colitis driven by the loss of NF- $\kappa \mathrm{B}$ essential modulator (NEMO) in the intestines. He also showed RIPK1 activation in samples from patients with psoriasis and rheumatoid arthritis. Overall, he highlighted the positive effects of inhibiting RIPK1 in these inflammatory diseases.

\section{Cell death in inflammatory diseases}

Francis KaMing Chan (Duke University, USA) demonstrated that while some viruses like Vaccinia virus sensitize cells to necroptosis, others inhibit it. In sum, different viruses have developed distinct strategies to escape from different types of cell death. Therefore the understanding of these strategies could result in novel therapeutic targets against viruses infections. Thirumala-Devi Kanneganti (St. Jude Children's Research Hospital, USA) demonstrated that ZBP1 and TAK1 are master regulators that drive pyroptosis, apoptosis, and necroptosis. Her findings revealed an opportunity to develop novel therapeutic strategies against infectious diseases, cancer and inflammation. Laura D. Attardi (Stanford University, USA) discussed the functions of p53 in suppressing tumor development and also the pathological roles of p53 in specific developmental abnormalities.

\section{Closing remarks}

The closing Keynote Address was presented by Shigekazu Nagata (Immunology Frontier Research Center, Japan). He reiterated Phosphatidylserine (PtdSer) exposition occurs in dead cells (efferocytosis) or living cells (entosis). Caspase-3 and caspase-7 cleave flippases and scramblases, activating and inactivating them, respectively, allowing PtdSer exposure on the surface of cells. He also demonstrated that besides caspase-mediated cleavage of flippases and scramblases, a kinase-mediated phosphorylation is also important for the exposure of PtdSer.

This Meeting was a milestone on cell death research. Novel mechanisms of action of crucial constituents of the machineries of several cell death pathways, such as caspase8 and GSDMD, were presented for the first time in public. We learned that cell death is a highly dynamic process with extensive plasticity. Therefore, manipulating one type of cell death can often result in the induction of another type of cell death and this may have notable pathophysiological consequences for the organisms. Future challenges are to develop novel therapeutic interventions, which must probably rely on targeting multiple molecules/cell death pathways in the treatment of inflammatory diseases, cancer and infectious diseases.

Acknowledgements We would like to thank all the speakers and attendees for making this symposium an outstanding meeting in the cell death field. We apologize for those interesting talks, workshop and poster presentations that could not be discussed here due to a limitation of space. We also thank the Keystone Symposia on Molecular and Cellular Biology, the Fundação de Amparo à Pesquisa do Estado de São Paulo (FAPESP), the Bill and Melinda Gates Foundation (BMGF) and Genentech, Inc. for their financial support of this conference.

\section{Compliance with ethical standards}

Conflict of interest The authors declare that they have no conflict of interest.

Publisher's note Springer Nature remains neutral with regard to jurisdictional claims in published maps and institutional affiliations.

\section{References}

1. Amarante-Mendes GP, Bortoluci KR, Bozza PT, Chammas R, Viola JPB. Paradise revealed: first-class science rocked by the sound of the waves. Cell Death Differ. 2010;17:1368-72. https:// doi.org/10.1038/cdd.2010.66.

2. Amarante-Mendes GP, Adjemian S, Mello BP, Hottz ED, Marques PE. Paradise revealed II: Top science, sunny beach, tropical fruits and caipirinhas. Cell Death Differ. 2015;22:1231-3. https://doi.org/ 10.1038/cdd.2014.242. 